\title{
Metastatic Breast Tumour Regression Following Treatment by a Gene-Modified Vaccinia Virus Expressing MUC1 and IL-2
}

\author{
Susy Scholl, ${ }^{1 *}$ Patrick Squiban, ${ }^{2}$ Nadine Bizouarne, ${ }^{2}$ Martine Baudin, ${ }^{2}$ Bruce Acres, ${ }^{2}$ Silvia von Mensdorff-Pouilly, ${ }^{3}$ \\ Moira Shearer, ${ }^{4}$ Philippe Beuzeboc, ${ }^{1}$ S. Van Belle, ${ }^{5}$ B. Uzielly, ${ }^{6}$ Pierre Pouillart, ${ }^{1}$ \\ Joyce Taylor-Papadimitriou, ${ }^{4}$ and David Miles ${ }^{4}$ \\ ${ }^{1}$ Institut Curie, Paris Cedex 05, France \\ ${ }^{2}$ Transgene S.A., Strasbourg, France \\ ${ }^{3}$ Free University Hospital, De Boelelaan 1117, 1081 HV, Amsterdam, Netherlands \\ ${ }^{4}$ Cancer Research UK Breast Cancer Biology Group, Guy's Hospital, London, UK \\ ${ }^{5}$ University Hospital, Gent, Belgium \\ ${ }^{6}$ Hadassah Hospital, Jerusalem, Israel
}

Received 28 May 2002; accepted 24 July 2002

\begin{abstract}
MUC1 is expressed by glandular epithelial cells. It is overexpressed in the majority of breast tumours, making it a potential target for immune therapy. The objectives of the present study were to evaluate the anti-tumour activity and tolerance of repeated administration of TG1031 (an attenuated recombinant vaccinia virus containing sequences coding for human MUC1 and the immune stimulatory cytokine IL-2) in patients with MUC1-positive metastatic breast cancer. This was an open-label, randomised study comparing two dose levels, $5 \times 10 \mathrm{E} 6$ and $5 \times 10 \mathrm{E} 7 \mathrm{pfu}$, with 14 patients in each arm. The treatment was administered intramuscularly every 3 weeks for the first 4 doses and every 6 weeks thereafter, until progression. Two patients had a partial tumour regression (>50\%), and 15 patients had stable disease as their best overall response until at least the 5th injection. Partial regression lasted for 11 months in one patient and for 12 months in the second patient who then underwent surgical resection of her hepatic metastases. The most frequent adverse events included inflammation at injection site: 7 patients, itching or pain at injection site: 5 patients, and moderate fever: 6 patients. One responding patient developed antinuclear, anti-DNA, and increased anti-TPO antibodies after the fifth injection, and which resolved at the end of treatment. The treatment regimes were well tolerated with a low toxicity profile. Although clinical efficacy remains limited, this study demonstrates the potential use of MUC1-based immune therapy in breast cancer.
\end{abstract}

\section{INTRODUCTION}

Reversal of tolerance and induction of antitumour activity has been shown following immunisation with fusions of dendritic and MUC1-positive carcinoma cells [1], and MUC1-positive CTL's have been detected following successful immunotherapy in renal cancer patients following administration of fused dendritic and renal cancer cells [2]. Mucins are large ( $>200 \mathrm{kd}$ ) glycoproteins with a high carbohydrate content ( $50-90 \%$ by weight). They are expressed by a variety of normal and malignant epithelial cells. MUC1 has a small transmembrane and intracellular tail and consists essentially of a large number (30-100) of repeated segments in tandem of 20 amino acids. The peptide core is densely coated with oligosaccharides, conferring a rigid rod-like structure which can extend several hundred nanometers from the apical cell surface into the lumen of ducts and glands. The biological function of MUC1 is thought to lubricate and protect glandular ducts, but knockout mice, lacking MUC1, had no alteration in their development and in their glandular morphology [3]. Mucins are attractive tumour antigens as they are overexpressed (10-40 fold) in a variety of cancers, making them useful targets for antibodies and cellular immunity. Cancer-associated MUC1 is incompletely glycosylated and as a result exposes internal sugar units and naked peptide sequences which are cryptic in the normal mucin molecule. MUC1 glycoprotein is often found in circulation in late-stage breast and lung cancer and used as a tumour marker (CA15.3) in the follow-up of breast cancer patients. Patients with high circulating MUC1 (CA153) serum levels have advanced cancer and are frequently in a state of T-cell unresponsiveness or anergy $[4,5]$. Although it had been suggested in the past that MUC1 caused Tcell unresponsiveness, a direct immunosuppressive role for MUC1 was excluded in more recent analyses [6].

The complete human MUC1 gene containing only 5 tandem repeats which had been inserted into a vaccinia 
virus vector, together with the gene coding for human interleukin 2, has been tested in a prior phase I study. A significant T-cell proliferative response against MUC1 following vaccination was observed in 1 of 9 advanced metastatic breast cancer patients. The stimulation index rose from 7 to 14 following a second injection. In addition, evidence of MUC1-specific CTL activity induced by VV-MUC1-IL-2 was seen. The present study was designed to expand on these earlier findings in metastatic breast cancer patients vaccinated after only one line of systemic treatment. Our objectives were to evaluate signs of clinical efficacy and tolerance of repeated injections as well as to assess the cellular and humoral response to MUC1 given at two different dose levels.

\section{PATIENTS AND METHODS}

\section{Patients}

We intended to enrol 28 patients into the study, 14 in each treatment group. The study was approved by each country's regulatory authorities and by the local ethical committees. The inclusion criteria were histologically proven MUC1-positive breast cancer, progressive and measurable metastatic disease following no more than one line of systemic treatment, WHO performance index $0-1$, and total lymphocyte counts greater than $0.75 \times 10$ E9/L. Principal exclusion criteria were recent viral infection, positive serology for HIV, major organ dysfunction, concomitant corticosteroid treatment, evidence of immunological compromise such as eczema or atopic dermatitis, and central nervous system metastasis. MUC1 antigen expression by the tumour was evaluated by immunohistochemistry using antibody H23 [7] on paraffin-embedded sections. In France, the immunological status of each patient was evaluated by a Pasteur-Mérieux multitest to recall antigens. Additional evaluations included antibodies to DNA and levels of serum amylase, $\beta 2$-microglobulin, C-reactive protein, total immunoglobulin levels, and CD4 : CD8 counts.

\section{Preclinical toxicology}

The human MUC1 and IL-2 cDNA were inserted in a head-to-head orientation under the control of the p7.5 promoter located at $5^{\prime}$ of each sequence. This block was inserted into the vaccinia virus (Copenhagen strain) genome. Previous results [8] demonstrated an attenuation of the viral virulence by both the inactivation of the viral TK as well as by the production of IL-2. Intracranial injection of this construct into nude mice was nontoxic, whereas injection of the parent strain was lethal in all instances (Bizouarne et al [9]). Transgenic mice expressing human MUC1 under the control of the human MUC1 promoter have been constructed and found to express MUC1 strongly and consistently in the cells lining the stomach. These mice had been used to assess, both histologically and macroscopically, any signs of autoim- munity induced by immunisation with VV-MUC1-IL-2 (VVTG-5058, the active viral ingredient of TG1031). No histological evidence of inflammatory cell infiltrate into normal tissues expressing MUC1 was observed, nor was there any gross evidence of autoimmune disease over a period of two months observation. Immunisation of these mice with MUC1 resulted in an anti-MUC1 antibody response which was dramatically lower than that seen in immunised nontransgenic mice, demonstrating a degree of tolerance to MUC1 at the level of antibody production. Nonetheless, MUC1-specific cytotoxic T lymphocyte precursor cells (CTL-pc) could be detected in both transgenic and nontransgenic mice following immunisation with VVTG-5058 [10].

Production of IL-2 by VVTG-5058-infected cells was measured both in vitro and in vivo. BHK-21 (baby hamster kidney) cells were infected with VVTG-5058 and the culture supernatant assessed for IL-2 both by ELISA and by bioactivity using the cell line CTLL-2. After 24-hour culture, the infected cells secretion of IL-2 ranging from $675 \mathrm{ng} / \mathrm{ml}$ to $1117 \mathrm{ng} / \mathrm{ml}$ and was equivalent to biologically active IL-2 ranging from 1283 to $8340 \mathrm{IU} / \mathrm{ml}$. Control supernatants from cells infected with the negative control wild-type virus contained no detectable IL-2. Production of IL-2 in vivo was assessed by injecting DBA/2 mice intramuscularly with VVTG-5058 and assessing IL-2 in the serum at later time points. Only at the 24-hour time point was IL-2 detectable at $50 \mathrm{pg} / \mathrm{ml}$ and it was thereafter undetectable. Viral dose was assessed by plaque forming activity on BHK cells.

\section{Patient monitoring}

Informed consent was obtained from each patient. $\mathrm{Pa}$ tients remained in a hospital room with a confinement compatible with HSE B2 specifications [11]. Patients were monitored in this confined room following each administration: for 24 hours after the first injection and for 2 hours after subsequent injections. In France, patients remained in this unit for 2 days on each occasion and until blood samples and nasopharyngeal swabs, taken on the day following the injection, were reported negative by PCR analysis. Vaccination with $0.5 \mathrm{ml}$ of TG1031 (doses of $5 \times 10 \mathrm{E} 6$ or $5 \times 10 \mathrm{E} 7 \mathrm{pfu}$ ) was done intramuscularly in the deltoid muscle.

\section{Viral monitoring (subgroup analysis)}

Samples were assessed for the presence of infectious vaccinia virus by PCR analysis at Texcell laboratories, Institut Pasteur, Paris. Special care was taken to prevent viral dissemination prior to and during transport. Swabs taken from the nose and throat, as well as samples of blood, were analysed prior to vaccination at day 0 and daily during the hospitalisation period. The detection limit was 2$9 \mathrm{pfu} / \mathrm{ml}$ in wet and $150 \mathrm{pfu} / \mathrm{ml}$ in dry samples. PCR analysis of virus was done with two pairs of primers designed to amplify either a $1.2-\mathrm{kbp}$ portion of a region of MUC1 with vaccinia flanking sequences or a second smaller region (740 bp) within MUC1. 


\section{Vaccinia- and MUC1-specific serology}

MUC1-specific IgG and IgM antibody levels were detected by ELISA with microtitre plates coated with a BSAconjugated MUC1 60mer peptide from the tandem repeat sequence as previously described [12].

\section{MUC1-specific proliferative responses by in vitro stimulation of PBMCs}

Ficoll/Hypaque separated peripheral blood mononuclear cells (PBMCs) were frozen in 10\% DMSO. Collected samples were thawed and tested for viability, and aliquots were incubated in the presence of mitogens (PHA and anti-CD3 monoclonal antibodies) and each antigen (human MUC1 from tandem repeat TAPPA-24 [13], mouse MUC1, purified protein derivative (PPD), and vaccinia virus) for 5 days. Cell proliferation is measured by the incorporation of ${ }^{3} \mathrm{H}$-thyminidine into the cellular DNA. Phytohemagglutinin (PHA), a mitogen which stimulates all T cells by cross-linking several cell surface glycoproteins by virtue of their sugar side chains, was used as a control. Another positive control mitogen, the anti-CD3 monoclonal antibody UCHT1, also was used.

\section{Cytokine production (subgroup analysis)}

Five hundred microlitre of the patients' plasma (lithium heparin) was aliquoted and stored at $-20^{\circ} \mathrm{C}$. All assays were carried out using commercial ELISA kits (R\&D, Minneapolis, Minn, USA) in duplicate samples and in the same assay testing for VEGF, M-CSF, TNF- $\beta 1$ as well as IL- 6 and IL-10. Results are expressed in pg/ml.

\section{RESULTS}

\section{Clinical summary}

Thirty-one female patients with metastatic breast cancer positive for MUC1 antigen were enrolled between June 1998 and March 1999 in four centres. Fourteen patients were randomised to the lower dose group $(5 \times 10 \mathrm{E} 6 \mathrm{pfu})$ and 17 patients to the higher dose group $(5 \times 10 \mathrm{E} 7 \mathrm{pfu})$. The patients' mean age was 56.3 years (ranging from 33 to 76 years), and the mean ECOG performance status at baseline 0.2 (ranging from 0 to 1 ).

Eight patients $(26 \%)$ had one metastatic site (3 patients $5 \times 10 \mathrm{E} 6 \mathrm{pfu}, 5$ patients $5 \times 10 \mathrm{E} 7 \mathrm{pfu}) ; 18$ patients $(58 \%)$ had $2-3$ metastatic sites (7 patients $5 \times$ $10 \mathrm{E} 6 \mathrm{pfu}, 11$ patients $5 \times 10 \mathrm{E} 7 \mathrm{pfu})$; and 5 patients had more than 3 metastatic sites ( 4 patients $5 \times 10 \mathrm{E} 6 \mathrm{pfu}$, 1 patient $5 \times 10 \mathrm{E} 7 \mathrm{pfu})$. All patients had received previous therapy for their metastatic breast cancer, including antioestrogens (24 patients), Goserelin (1), aromatase inhibitors (10), anthracyclines [alone (8) or in combination with (FEC/FAC) (9)], Taxotere/Taxol (8), 5FU-based chemotherapy (8), MMM (2), and Navelbine (4). One patient (patient 106, $5 \times 10 \mathrm{E} 7 \mathrm{pfu}$ group) had received prior immunotherapy. Patients received a median number of 3 cycles (ranging from 2 to 8 ) of drug treatment during the study $[5 \times 10 \mathrm{E} 6 \mathrm{pfu}$ group: median of 4 cycles (ranging
TABle 1. Tumor measurements in patient 207.

\begin{tabular}{ccccc}
\hline Liver lesion & January 11 & March 3 & May 7 & August 6 \\
\hline Segment VII & $19 \times 19$ & $18 \times 18$ & $16 \times 16$ & $11 \times 11$ \\
\hline Segment VI & $28 \times 24$ & $28 \times 22$ & $26 \times 22$ & $19 \times 19$ \\
\hline Cupole & $20 \times 20$ & $15 \times 20$ & $12 \times 14$ & $4 \times 4$ \\
\hline
\end{tabular}

from 2 to 7 ) and $5 \times 10 \mathrm{E} 7$ pfu group: median of 3 cycles (ranging from 2 to 8 )].

\section{Efficacy analysis}

Two out of $31(6 \%)$ patients achieved a partial response (PR), 1 in each treatment group. No complete response was seen. Stable disease (SD) was the best overall response observed in $15(48 \%)$ patients ( 7 in the $5 \times$ $10 \mathrm{E} 6 \mathrm{pfu}$ group and 8 in the $5 \times 10 \mathrm{E} 7 \mathrm{pfu}$ group).

Patient $207(5 \times 10$ E6 pfu treatment group) was treated for over 1 year (11 cycles) with PR then SD first detected on day 148. She had a surgical resection of the remaining liver metastases and was free of disease at the time of analysis, 18 months after the start of vaccination (Table 1 ). Patient $204(5 \times 10 \mathrm{E} 7 \mathrm{pfu}$ treatment group $)$ was treated for 9 cycles, showing a PR on day 103, and remains stable at 11 months of treatment. This patient's tumour regressed subsequently with perfusion chemotherapy.

\section{Time to progression}

The median time to progression in the $5 \times 10 \mathrm{E} 6 \mathrm{pfu}$ group was 91 days (95\% confidence interval (CI): 35 to 105 days), compared to 59 days ( $95 \%$ CI: 38 to 81 days) in the $5 \times 10 \mathrm{E} 7$ pfu group (NS). A total of 7 patients $(23 \%)$ died, one (patient $109,5 \times 10 \mathrm{E} 6$ pfu group) because of progressive disease during the course of the study, 5 weeks after the start of treatment. Six patients died of progressive disease during the follow-up period, defined as more than 28 days after last injection $(1$ in the $5 \times 10 \mathrm{E} 6$ pfu group and 5 in the $5 \times 10$ E7 pfu group).

\section{Safety}

All the 31 patients enrolled in the study were included in the safety analysis. The administration of TG1031 was generally well tolerated. All patients experienced at least one adverse event. Adverse events were considered as treatment-related in $22 / 31$ (71\%) of patients $(5 \times 10 \mathrm{E} 6 \mathrm{pfu}$ group: 9 patients; $5 \times 10 \mathrm{E} 7 \mathrm{pfu}$ group: 13 patients). The most frequently reported adverse events were general disorders and reactions at the injection site in 23/31 (74\%) of patients $(5 \times 10 \mathrm{E} 6$ pfu group: 8 patients and $5 \times 10 \mathrm{E} 7 \mathrm{pfu}$ group: 15 patients) followed by gastrointestinal disorders in 15 patients $(5 \times 10 \mathrm{E} 6$ pfu group: 7 patients; $5 \times 10 \mathrm{E} 7 \mathrm{pfu}$ group: 8 patients) and musculoskeletal and bone disorders in 14 patients $(5 \times 10 \mathrm{E} 6 \mathrm{pfu}$ group: 6 patients; $5 \times 10$ E7 pfu group: 8 patients). Only one patient (patient $305,5 \times 10$ E7 pfu group) had a grade- 3 fatigue which was considered as probably drug-related and which resolved 
within 14 days. Five patients reported serious adverse events $(5 \times 10 \mathrm{E} 6$ pfu group: 2 patients; $5 \times 10 \mathrm{E} 7$ pfu group: 3 patients) that were considered unrelated to study medication $(5 \times 10 \mathrm{E} 6$ pfu group: 2 patients had dyspnoea and worsening of preexisting dyspnoea; $5 \times 10 \mathrm{E} 7 \mathrm{pfu}$ group: 3 patients had vaginal haemorrhage/anaemia, worsening of preexisting dyspnoea, and fever-due to indwelling catheter). None of the NCIC-CTC (National Cancer Institute of Canada-Common Toxicity Criteria) grade 3/4 abnormal laboratory values were considered clinically significant.

\section{Viral dissemination}

Tests for viral dissemination were carried out as described in material and methods. All viral tests were consistently negative.

\section{Biological results}

\section{T-cell proliferation assays}

\section{Cellular immune response to the vector}

Cellular immune responses were measured by culturing patients' PBMCs together with one of a panel of several antigens and mitogens. For all patients, the PBMCs responded normally to UCHT1, an anti-CD3 antibody. PPD from the tuberculin bacillus was used as a positive control antigen. Most Europeans have been immunised with tuberculin during their lifetime and most patients' PBMCs responded normally to PPD, with some variation throughout the study. Reactivity to UV-inactivated VV particles was equally assessed. Despite the fact that most, if not all patients, had received smallpox vaccinations during their lifetime, PBMCs from only one patient (patient 106) showed T-cell proliferation to VV at baseline. PBMCs from most, but not all patients, proliferated in response to culture with VV at the second and subsequent injection time points. There was a significant variability in the condition of the cells and their ability to proliferate in response to antigen. Negative controls were either no antigen at all or MUC1 tandem repeat peptide from the murine MUC1 sequence, which has little or no cross-reactivity with human MUC1.

\section{Cellular immune response to MUC1}

None of the PBMCs from patients in this study showed any significant $\mathrm{T}$-cell proliferation in response to a 24-amino acid polypeptide corresponding to one MUC1 variable-number tandem repeat sequence. Nevertheless, PBMCs from patients 207 and 208, for example, at some time points were able to respond to both PPD and VV antigens without evidence of proliferation in response to the MUC1 antigen by the same PBMCs. The interpretation, therefore, is that there was no evident systemic Tcell response to the tandem repeat (TAPPA 24) peptide of MUC1 by PBMCs from the patients in this study as detected by this method.

\section{Vaccinia-and MUC1-specific antibodies}

\section{Antibody responses to the vector (vaccinia virus)}

Most patients showed evidence of preexisting antibody immunity to vaccinia virus. All patients showed increased anti-VV titres after vaccination, with the exception of patient 202 who had a comparatively high VVspecific IgG titre in her pre-inclusion plasma sample, which did not change throughout the course of TG1031 vaccinations, of which she received six. This suggests that the patient had received a smallpox vaccination, but failed to generate a recall response to TG1031. The most pronounced rise in titre occurred in those patients receiving the highest dose.

\section{Antibody responses to MUC1}

The analysis of MUC1-specific antibody titres was performed according to a published method [14]. There was no significant antibody response to MUC1 upon immunotherapy with TG1031, and levels of antibody did not exceed levels found in some cancer patients. There were few notable fluctuations: MUC1-specific IgG levels in plasma from patient 104 dropped to $50 \%$ of pretreatment levels at cycles 4 and 5 then increased to levels slightly higher than baseline in subsequent samples. This was not thought to be related to levels of complex formation with circulating MUC1, since CA-15.3 levels increased only slightly in this patient and remained within the normal range. A similar phenomenon had been observed in one patient in the VV-MUC1-IL-2 phase I clinical trial. Small increases of IgG MUC1-specific antibody titres were detected in four patients, including the clinical responders.

\section{CD4 levels and outcome (subgroup analysis)}

In view of the reported absence of toxicity in animal studies and the reported attenuation by viral TK inactivation [15] and local IL-2 production [16], no restrictions on inclusion by number of CD4+ lymphocyte counts had been planned and this information is only available from the subgroup analysis in France. CD4 counts immediately preceding vaccinations were consistently above 600 in 4 patients including the 2 partial responders, reaching values as high as 1400 counts $/ \mathrm{mm}^{3}$ after several injections while CD4 levels in patients with rapidly progressive disease were low at baseline and did not increase after several injections (Figure 1).

\section{Cytokine plasma levels (subgroup analysis)}

Cytokines that have repeatedly been associated with immune suppression or defective dendritic cell differentiation are shown in Figure 1 and Table 2.

Prevaccination M-CSF levels were the highest (669 and $957 \mathrm{pg} / \mathrm{ml}$ ) in patients 203 and 206 who progressed early and the lowest ( 235 and $246 \mathrm{pg} / \mathrm{ml}$ ) in patients 204 and 207 who developed a clinical response. ELISA assays using the same kit in over 600 primary breast cancers prior to treatment had shown median values of $380 \mathrm{pg} / \mathrm{ml}$. 


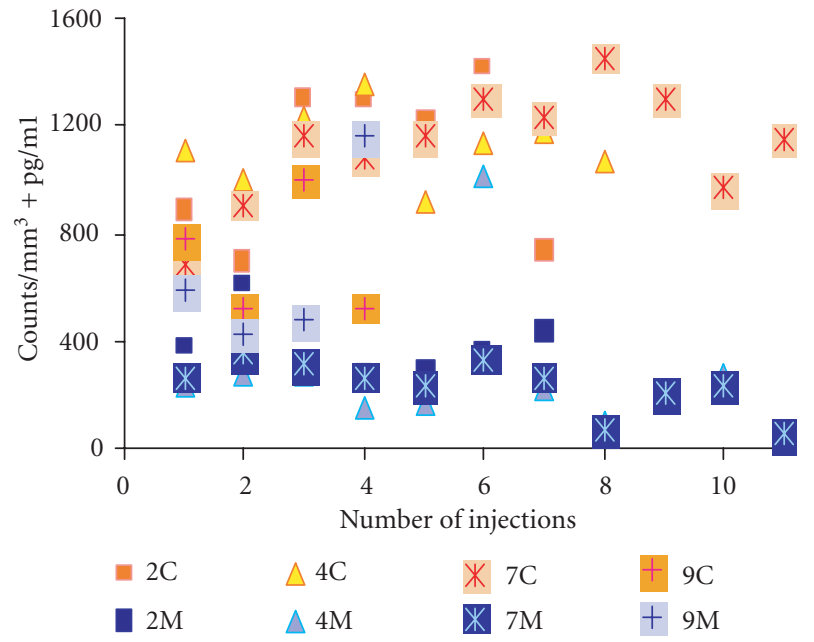

(a) Patients with baseline high CD4 and low M-CSF levels: PR in patients 204 and 207.

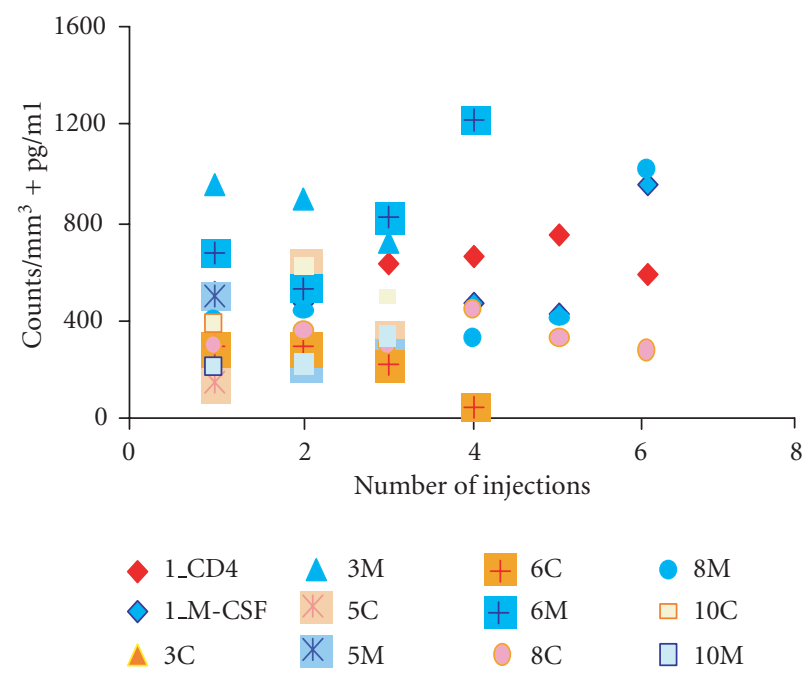

(b) Patients with baseline low CD4 and/or high M-CSF levels.

FIGURe 1

Both patients who showed a partial clinical response had consistently low M-CSF levels and high CD4 counts. No specific patterns in other serum cytokines (IL-10, TGFb1, or VEGF) were detected.

\section{Autoimmunity}

Signs of autoimmunity were detected in 3 out of 10 patients following either routine screening for anti-DNA antibodies (patient 207), following subsequent retrospective screening for anti-TPO (thyro-peroxidase) antibodies (patients 205 and 207), or following biopsy of accessory salivary gland for dry mouth (patient 209).

Patient 207, who showed the best response to study treatment, developed high titres of anti-DNA antibodies (up to $15 \mathrm{U} / \mathrm{ml}$ after 6 injections) and raised anti-TPO
TABLE 2. Immunosuppressive cytokines.

\begin{tabular}{|c|c|c|c|c|}
\hline & & VEGF & TGF b1 & rec IL-10 \\
\hline \multirow[t]{3}{*}{1} & base line & 0 & 434 & 0 \\
\hline & 3 & 21 & 338 & 0 \\
\hline & 5 & 54 & 1610 & 0 \\
\hline \multirow[t]{4}{*}{2} & base line & 143 & 326 & 0 \\
\hline & 3 & 216 & 1520 & 0 \\
\hline & 4 & 41 & 572 & 0 \\
\hline & 6 & 342 & 170 & 0 \\
\hline \multirow[t]{4}{*}{3} & base line & 173 & 644 & 0 \\
\hline & 1 & 39 & 2402 & 0 \\
\hline & 2 & 66 & 1256 & 0 \\
\hline & 3 & 41 & 506 & 0 \\
\hline \multirow[t]{4}{*}{4} & base line & 136 & 2450 & 0 \\
\hline & 3 & 301 & 164 & 0 \\
\hline & 5 & 78 & 548 & 0 \\
\hline & 8 & 328 & 2678 & 0 \\
\hline \multirow[t]{2}{*}{5} & base line & 738 & 440 & 0 \\
\hline & 3 & 236 & 6170 & 0 \\
\hline \multirow[t]{2}{*}{6} & 3 & 187 & 950 & 0 \\
\hline & end & 276 & 692 & 57 \\
\hline \multirow[t]{4}{*}{7} & base line & 43 & 164 & 0 \\
\hline & 3 & 36 & 836 & 0 \\
\hline & 4 & 72 & 2408 & 0 \\
\hline & 8 & 218 & 962 & 0 \\
\hline \multirow[t]{3}{*}{8} & base line & 44 & 272 & 0 \\
\hline & 3 & 10 & 266 & 0 \\
\hline & 5 & 9 & 482 & 0 \\
\hline 9 & 3 & 20 & 272 & 0 \\
\hline \multirow[t]{2}{*}{10} & base line & 30 & 266 & 0 \\
\hline & 2 & 85 & 1628 & 0 \\
\hline
\end{tabular}

antibodies (baseline: $179 \mathrm{U} / \mathrm{ml}$; extremes: $11529 \mathrm{U} / \mathrm{ml}$ after the 8th injection down to $1438 \mathrm{U} / \mathrm{ml}$ five months after the 10th injection for a normal range $<60 \mathrm{U} / \mathrm{ml}$ ). Circulating CD4 lymphocyte numbers doubled prior to the third injection, peaking at $1444 \times 10^{6} / \mathrm{L}$ at the 8 th injection (CD4/CD8 ratio: 5.5) at a time point consistent with a response to treatment (Table 3). Thyroid function tests were subnormal in one occasion (after the 8th injection) to return to the normal range within 3 weeks. The patient had no clinical signs of thyroid insufficiency. Renewed questioning revealed a family history of thyroid disorder. One sister also had breast cancer. These biological signs of autoimmunity spontaneously resolved while the patient was still being treated.

\section{DISCUSSION}

The use of vaccinia virus as a carrier and an adjuvant for overexpressed tumour antigens has been reported in a number of phase I trials in a variety of patients with 
TABLE 3. Variations of CD4 levels and antithyroid antibodies over the course of treatment in patient 207.

\begin{tabular}{|c|c|c|c|c|c|c|c|c|c|}
\hline injection \# & date & CD4 & $\mathrm{CD} 4 / \mathrm{CD} 8$ & CA153 & anti-TPO & anti-nuclear & anti-DNA & $\mathrm{T} 4$ & TSH \\
\hline & & counts $/ \mathrm{mm}^{3}$ & ratio & $\mathrm{U} / \mathrm{ml}$ & $\mathrm{U} / \mathrm{ml}$ & Inverse ratio & $\mathrm{U} / \mathrm{ml}$ & $\mathrm{ng} / \mathrm{L}$ & $\mu \mathrm{U} / \mathrm{ml}$ \\
\hline $\mathrm{BL}$ & $20 / 01 / 99$ & 680 & & 26 & 179 & 0 & 0 & & \\
\hline 1 & $28 / 01 / 99$ & & & 23 & & 0 & 0 & 10.7 & 1.18 \\
\hline 2 & $18 / 02 / 99$ & 908 & & 18 & & 0 & 0 & 10.3 & 1.94 \\
\hline 3 & $11 / 03 / 99$ & 1160 & 4.7 & 18 & & 0 & 0 & & \\
\hline 4 & $01 / 04 / 99$ & 1081 & 5.2 & 17 & & 0 & 0 & & \\
\hline 5 & $17 / 05 / 99$ & 1172 & 5.6 & 16 & & 0 & 0 & 12 & 2.92 \\
\hline 6 & $28 / 06 / 99$ & 1305 & & 18 & & 80 & 14 & 15.2 & 2.23 \\
\hline 7 & $09 / 08 / 99$ & 1224 & 4.7 & 17 & & 160 & 15 & & \\
\hline$\underline{8}$ & $20 / 09 / 99$ & $\underline{1444}$ & $\underline{5.5}$ & $\underline{18}$ & 11529 & $\underline{320}$ & $\underline{13}$ & $\underline{5.8}$ & $\underline{51.29}$ \\
\hline 9 & $02 / 11 / 99$ & 1345 & 3.5 & 18 & 11052 & & & 11.9 & 9.14 \\
\hline 10 & $13 / 12 / 99$ & 966 & 4.7 & 18 & 6667 & 260 & 7 & 12 & 0.97 \\
\hline
\end{tabular}

solid tumours such as breast, prostate, and cervical cancer $[17,18,19]$. Vaccinations of wild life against rabies have shown vaccinia virus to be effective and safe for the environment [20]. Nonpathogenic viruses for humans, such as fowl pox viruses, are also used in vaccination trials and have been shown to increase the efficacy of boost injections in alternate prime and boost strategies using different vectors with different antigenic epitopes [12]. We have developed a recombinant vaccinia virus, replicative but attenuated, that expresses upon infection the widely overexpressed breast cancer antigen MUC1 as well as the human immunostimulatory cytokine IL-2. We have been able to confirm the safety and efficacy of repeated injections of the same vector as well as provide clinical evidence of vaccine efficacy in metastatic breast cancer patients.

In the present phase II study, repeated injections of this vector in patients with less advanced disease generated clinical regressions of axillary lymph nodes (seen after 1 injection) and of liver metastases (after 3 injections) (Figure 1). Increasing evidence exists from murine, primate, and human studies to confirm that T-cell proliferative responses as well as MHC-restricted cytotoxic T cells can be induced by immunisation with MUC1 antigenic epitopes $[4,21,22,23,24,25,26,27,28]$. In the present study, no specific proliferative response directed against an epitope of the human MUC1 tandem repeat domain could be detected in any of our patients. Although with the same assay T-cell proliferation was seen in a previous trial [3], the proof of treatment-related MUC1specific systemic cellular immune responses could not so far be confirmed in the present patients by in vitro testing. The recent demonstration of class I HLA-A2 restricted epitopes derived from the MUC-1 tandem repeat region or from the leader sequence of MUC-1 suggests that the MUC-1-directed immune responses may not be limited to the extracellular tandem repeat domain [26]. Using peptide-pulsed dendritic cells in addition to Pan-HLA-DR binding peptides, Brossart et al [26] also demonstrated the importance of CD4 cells for efficient CTL priming. It remains to be seen whether the patients who demonstrated a clinical response developed CTL's or a proliferative response against other epitopes that we have not yet tested for. Murine experiments have shown that immunisation with MUC1 without tandem repeats is as effective as immunisation with the entire MUC1 in inducing a tumour rejecting anti-MUC1 immune response (Taylor-Papadimitriou et al [29]). In addition and in a separate study, several patients immunised with MUC1 showed T-cell proliferative responses to MUC1 antigenic epitopes outside the tandem repeat region (Rochlitz et al [30]).

All but one patient had serological evidence of vaccination with vaccinia virus in childhood. Antibodies against the virus were detectable prior to vaccination, and patients treated with the highest dose levels had increased serological responses to vaccinia virus, but surprisingly little response to MUC1. Our results are in contrast with those of Apostolopoulos et al [30] who document a predominant antibody response following serial vaccinations with a peptide-mannan fusion protein. High titres of antibodies to MUC1 in MUC1-immunised patients are possible, as reported by other clinical trials in which patients had been immunised with polypeptides from the MUC1 tandem repeat sequence [31]. Antibody, but not T-cell tolerance to MUC1 has been observed in MUC1 transgenic mice immunised with vaccinia-MUC1-IL-2 [10]. It is not clear whether antibodies may give equivalent antitumour protection to that of a cellular immune response. A benefit in survival has been observed in early-stage breast cancer patients with elevated natural antibodies to MUC1 [20]. Similarly, an increase in antibody titres has been reported in multiparous women [13], and Agrawal et al speculated that the well-known decreased breast cancer risk in women having pregnancies early in their reproductive life might be related to spontaneous vaccination against this breast tumour antigen. Only one patient (number 301) injected with VV-MUC1-IL-2 had a notable antibody titre 
to MUC1 prior to vaccination and maintained significant titres of IgM anti-MUC1 antibodies following vaccination. Vaccination using the complete MUC1 gene and therefore the presentation of a variety of MUC1 class I or II epitopes in the context of a viral expression vector may also influence the type (cellular or humoral) of response.

We did not observe any virus-related toxicity, nor did we detect any viral particles by PCR technology, suggesting that the repetitive viral injection was safe. Antiviral antibodies were present and increased mildly during treatment.

In a subgroup analysis, a rise in circulating (nonspecified) CD4 $\mathrm{T}$ lymphocyte counts was seen in some patients, and in particular, in the two responding patients suggestive of that the immune system still susceptible to stimulation. These results compare with observations from the literature showing a correlation between high level of circulating $\mathrm{CD} 4_{+}$lymphocytes and the favourable outcome. Recent evidence from a human melanoma model suggested that the true benefit of $\mathrm{CD} 4_{+} \mathrm{T}$ cells may be not only in the induction and maintenance of cytotoxic $\mathrm{T}$ lymphocytes, but possibly in a direct (CD4-mediated) antitumour response [33]. Low levels of immune-suppressive cytokines such as M-CSF, TGF $\beta 1$, IL-6, VEGF, and low C-reactive protein had been seen in those patients able to develop CTL's against MUC1 following vaccination in the past [23]. High amounts of M-CSF, VEGF, and IL-6 in tumour cell supernatants have been associated with suppression of dendritic cell differentiation [34, 35] as well as with tumour progression. Tumour-generated suppressive factors may thus represent a limiting factor to immunotherapy. In primary breast carcinomas, Bell et al did detect only immature dendritic cells within the tumour, whereas mature dendritic cells were located in peritumoral areas [36].

In the same subgroup, several low-grade autoimmune manifestations were documented in 3 of 10 patients. The rise in self antibodies was parallel to the rise in peripheral CD4 cells and to the clinical response in one patient. The presence of inflammatory cells in a biopsy of accessory salivary glands was seen in one patient who had complained of dry mouth following injection. Autoimmune reactions have also been documented in renal cell cancers following treatment with IL-2 [37]. The patients with signs of antithyroid antibodies had a significantly better survival, suggesting that a mild degree of autoimmunity may be a positive sign more than an "acceptable side effect" of antitumour vaccinations.

In conclusion, multiple injections of VV-MUC1-IL-2 were clinically well tolerated and able to induce clinical responses in two patients. Mild autoimmune effects were present in some patients, including one of the clinical responders. We suggest monitoring peripheral CD4 titres and autoantibodies in future trials.

\section{REFERENCES}

[1] Gong J, Chen D, Kashiwaba M, et al. Reversal of tolerance to human MUC1 antigen in MUC1 transgenic mice immunized with fusions of dendritic and carcinoma cells. Proc Natl Acad Sci USA. 1998;95(11):6279-6283.

[2] Kugler A, Stuhler G, Walden P, et al. Regression of human metastatic renal cell carcinoma after vaccination with tumor cell-dendritic cell hybrids. Nat Med. 2000;6(3):332-336.

[3] Spicer AP, Rowse GJ, Lidner TK, Gendler SJ. Delayed mammary tumor progression in Muc-1 null mice. $J$ Biol Chem. 1995;270(50):30093-30101.

[4] Agrawal B, Gendler SJ, Longenecker BM. The biological role of mucins in cellular interactions and immune regulation: prospects for cancer immunotherapy. Mol Med Today. 1998;4(9):397-403.

[5] Agrawal B, Krantz MJ, Reddish MA, Longenecker BM. Cancer-associated MUC1 mucin inhibits human T-cell proliferation, which is reversible by IL-2. Nat Med. 1998;4(1):43-49.

[6] Paul S, Bizouarne N, Paul A, et al. Lack of evidence for an immunosuppressive role for MUC1. Cancer Immunol Immunother. 1999;48(1):22-28.

[7] Keydar I, Chou CS, Hareuveni M, et al. Production and characterization of monoclonal antibodies identifying breast tumor-associated antigens. Proc Natl Acad Sci USA. 1989;86(4):1362-1366.

[8] Buller RM, Smith GL, Cremer K, Notkins AL, Moss B. Decreased virulence of recombinant vaccinia virus expression vectors is associated with a thymidine kinase-negative phenotype. Nature. 1985;317(6040):813-815.

[9] Bizouarne N, Balloul J-M, Schatz C, Acres B, Kieny M-P. Immunotherapy of Breast Cancer with a recombinant Vaccinia virus expressing the human MUC1 and IL2. In: Calvo F, Crepin M, Magdelenat $\mathrm{H}$, eds. Breast Cancer, Advances in Biology and Therapeutics. Montrouge: John Libby Eurotext; 1996:303308.

[10] Acres B, Apostolopoulos V, Balloul JM, et al. MUC1-specific immune responses in human MUC1 transgenic mice immunized with various human MUC1 vaccines. Cancer Immunol Immunother. 2000;48(10):588-594.

[11] Health \& Safety Executive. A Guide to the Genetically Modified Organisms (contained use) Regulations. Subdury UK: Health \& Safety Executive; 1996.

[12] Hodge JW, McLaughlin JP, Kantor JA, Schlom J. Diversified prime and boost protocols using recombinant vaccinia virus and recombinant nonreplicating avian pox virus to enhance T-cell immunity and antitumor responses. Vaccine. 1997;15(67):759-768.

[13] Agrawal B, Reddish MA, Krantz MJ, Longenecker BM. Does pregnancy immunize against breast cancer? Cancer Res. 1995;55(11):2257-2261. 
[14] von Mensdorff-Pouilly S, Gourevitch MM, Kenemans $\mathrm{P}$, et al. An enzyme-linked immunosorbent assay for the measurement of circulating antibodies to polymorphic epithelial mucin (MUC1). Tumour Biol. 1998;19(3):186-195.

[15] Buller RM, Smith GL, Cremer K, Notkins AL, Moss B. Decreased virulence of recombinant vaccinia virus expression vectors is associated with a thymidine kinase-negative phenotype. Nature. 1985;317(6040):813-815.

[16] Flexner C, Hugin A, Moss B. Prevention of vaccinia virus infection in immunodeficient mice by vector-directed IL-2 expression. Nature. 1987; 330(6145):259-262.

[17] Sanda MG, Smith DC, Charles LG, et al. Recombinant vaccinia-PSA (PROSTVAC) can induce a prostate-specific immune response in androgenmodulated human prostate cancer. Urology. 1999; 53(2):260-266.

[18] Eder JP, Kantoff PW, Roper K, et al. A phase I trial of recombinant prostate specific antigen expressing vaccinia virus vaccine, PROSTVAC (rV-PSA) in advanced Prostate. In: Cancer Americans Society of Clinical Oncology, Annual Meeting; 1999.

[19] Borysiewicz LK, Fiander A, Nimako M, et al. A recombinant vaccinia virus encoding human papillomavirus types 16 and 18, E6 and E7 proteins as immunotherapy for cervical cancer. Lancet. 1996;347(9014):1523-1527.

[20] Brochier B, Kieny MP, Costy F, et al. Large scale eradication of rabies using recombinant mucin vector: in vitro analysis of expression of tumor-associated epitopes for antibody and human cytotoxic T-cell recognition. J Immunother. 1993;14:127-135.

[21] Apostolopoulos V, Karanikas V, Haurum JS, McKenzie IF. Induction of HLA-A2-restricted CTLs to the mucin 1 human breast cancer antigen. J Immunol. 1997;159(11):5211-5218.

[22] Acres RB, Hareuveni M, Balloul JM, Kieny MP. Vaccinia virus MUC1 immunization of mice: immune response and protection against the growth of murine tumors bearing the MUC1 antigen. J Immunother. 1993;14(2):136-143.

[23] Scholl SM, Balloul JM, Le Goc G, et al. Recombinant vaccinia virus encoding human MUC1 and IL2 as immunotherapy in patients with breast cancer. $J$ Immunother. 2000;23(5):570-580.

[24] Mukherjee P, Madsen C, Canales N, Sterner CJ, Hollingsworth MA, Gendler SJ. MUC-1 specific CTLs detected in human MUC1 transgenic mice that spontaneously develop pancreatic tumors. Proc AACR 3113A, 1999.

[25] Apostolopoulos V, Karanikas V, Haurum JS, McKenzie IF. Induction of HLA-A2-restricted CTLs to the mucin 1 human breast cancer antigen. J Immunol. 1997;159(11):5211-5218.

[26] Brossart P, Heinrich KS, Stuhler G, et al. Identification of HLA-A2-restricted T-cell epitopes derived from the MUC1 tumor antigen for broadly applicable vaccine therapies. Blood. 1999;93(12):43094317.

[27] Barratt-Boyes SM, Vlad A, Finn OJ. Immunization of chimpanzees with tumor antigen MUC1 mucin tandem repeat peptide elicits both helper and cytotoxic T-cell responses. Clin Cancer Res. 1999;5(7):1918-1924.

[28] Reddish M, MacLean GD, Koganty RR, et al. AntiMUC1 class I restricted CTLs in metastatic breast cancer patients immunized with a synthetic MUC1 peptide. Int J Cancer. 1998;76(6):817-823.

[29] Taylor-Papadimitriou J, Burchell JM, Plunkett T, et al. MUC1 and the immunobiology of cancer. J Mammary Gland Biol Neoplasia. 2002;7(2):209221.

[30] Rochlitz C, Figlin R, Squiban P, et al. Phase I immunotherapy with a modified vaccinia virus (MVA) expressing human MUC1 as antigen-specific immunotherapy in patients with MUC1-positive advanced cancer. The Journal of Gene Medicine. In press.

[31] Apostolopoulos V, Osinski C, McKenzie IF. MUC1 cross-reactive Gal alpha $(1,3) \mathrm{Gal}$ antibodies in humans switch immune responses from cellular to humoral. Nat Med. 1998;4(3):315-320.

[32] Karanikas V, Hwang LA, Pearson J, et al. Antibody and $\mathrm{T}$ cell responses of patients with adenocarcinoma immunized with mannan-MUC1 fusion protein. J Clin Invest. 1997;100(11):2783-2792.

[33] Zarour HM, Kirkwood JM, Kierstead LS, et al. Melan-A/MART-1(51-73) represents an immunogenic HLA-DR4-restricted epitope recognized by melanoma-reactive CD4(+) T cells. Proc Natl Acad Sci USA. 2000;97(1):400-405.

[34] Gabrilovich DI, Chen HL, Girgis KR, et al. Production of vascular endothelial growth factor by human tumors inhibits the functional maturation of dendritic cells. Nat Med. 1996;2(10):1096-1103.

[35] Menetrier-Caux C, Montmain G, Dieu MC, et al. Inhibition of the differentiation of dendritic cells from CD34(+) progenitors by tumor cells: role of interleukin-6 and macrophage colony-stimulating factor. Blood. 1998;92(12):4778-4791.

[36] Bell D, Chomarat P, Broyles D, et al. In breast carcinoma tissue, immature dendritic cells reside within the tumor, whereas mature dendritic cells are located in peritumoral areas. J Exp Med. 1999;190(10):14171426.

[37] Franzke A, Peest D, Probst-Kepper M, et al. Autoimmunity resulting from cytokine treatment predicts long-term survival in patients with metastatic renal cell cancer. J Clin Oncol. 1999;17(2):529-533.

\footnotetext{
* Corresponding author.

E-mail: sscholl@curie.net
}

Fax: +33 1443246 87; Tel: +33144324671 


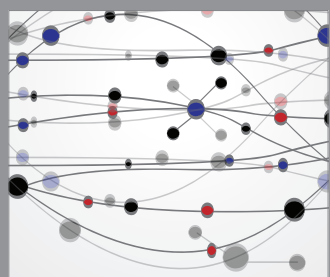

The Scientific World Journal
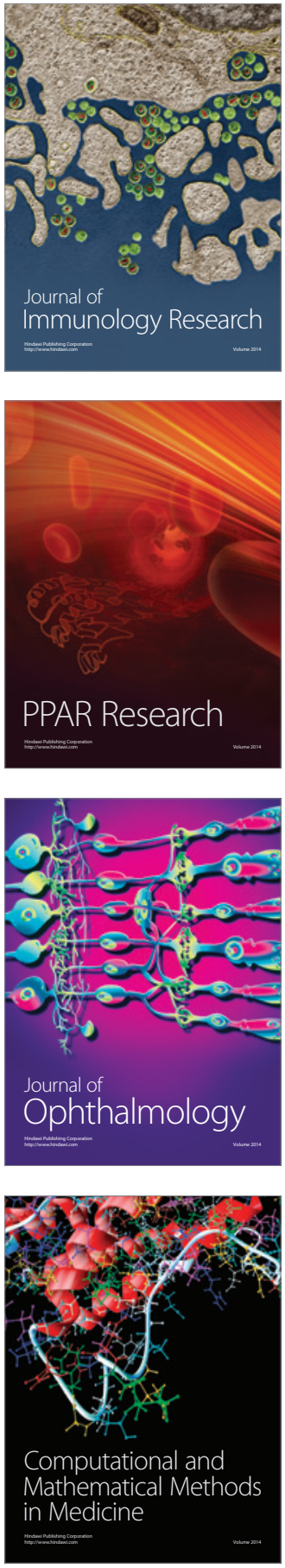

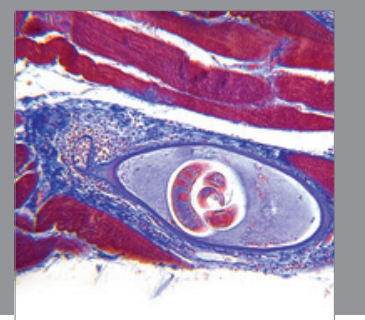

Gastroenterology

Research and Practice
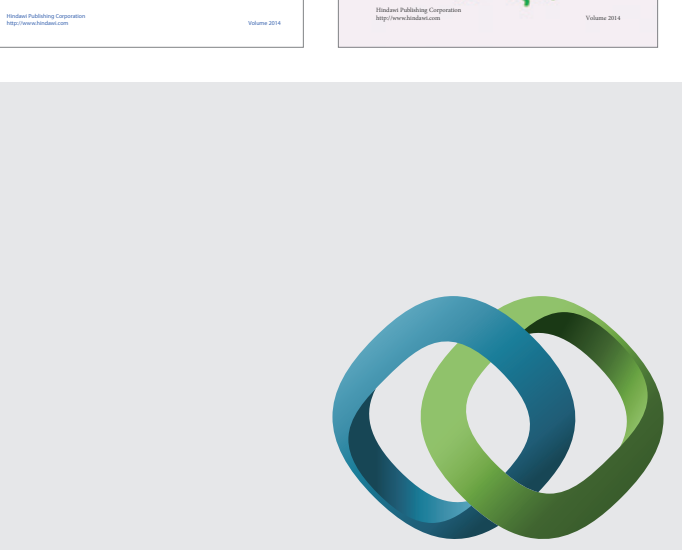

\section{Hindawi}

Submit your manuscripts at

http://www.hindawi.com
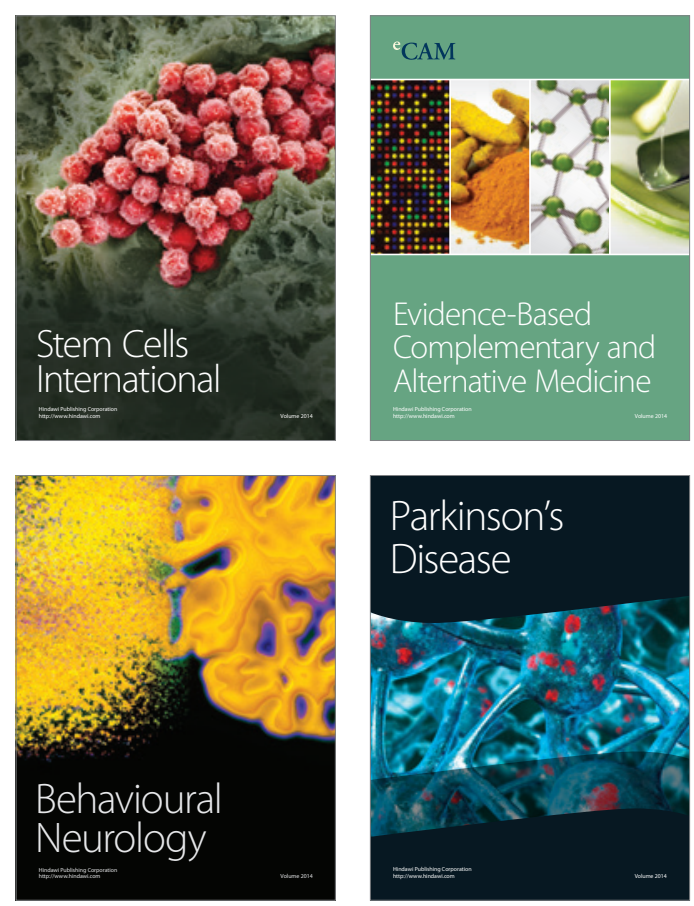

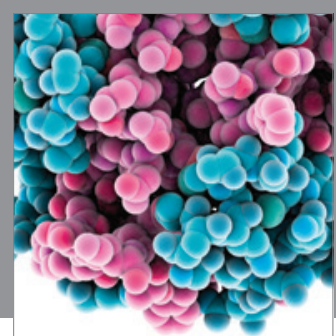

Journal of
Diabetes Research

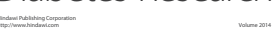

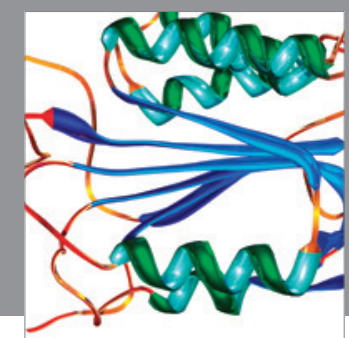

Disease Markers
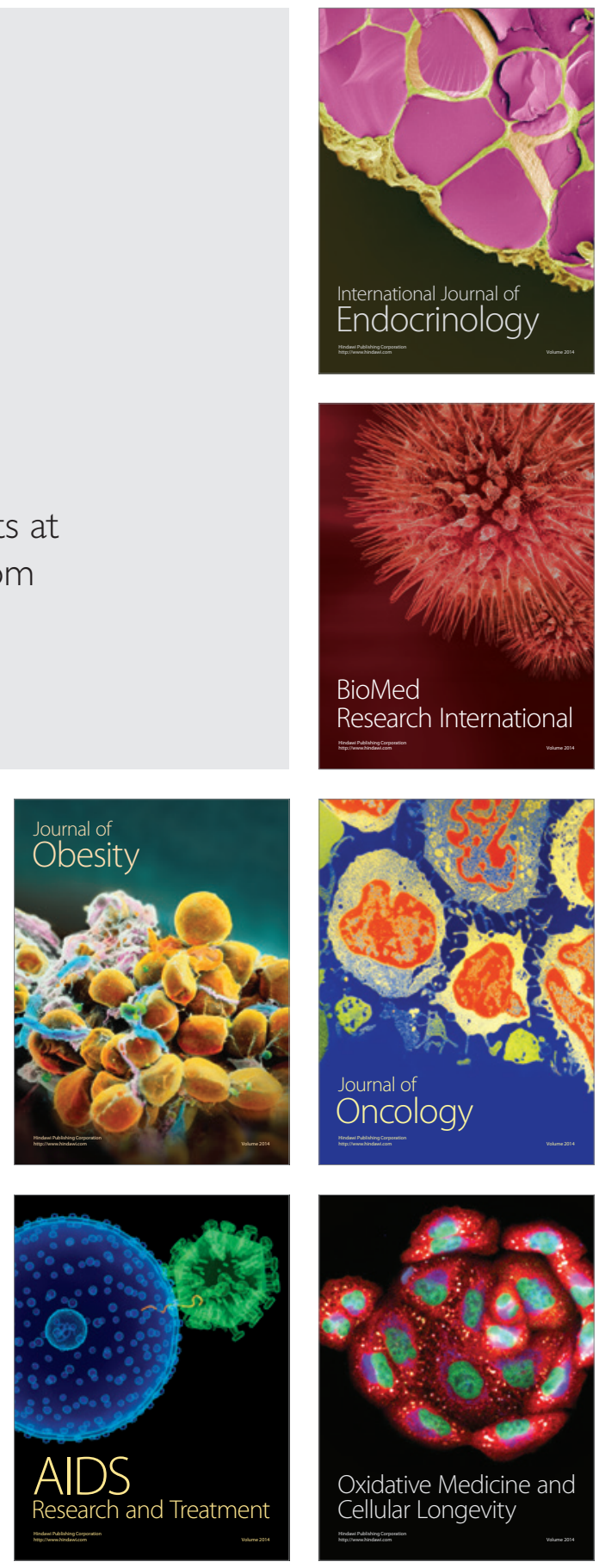